\title{
Successful Treatment of Severely Hypotensive Pediatric Patients with Multisystem Inflammatory Syndrome in Children (MIS-C) with the Guidance of Invasive Hemodynamic Monitoring: A Report of Three
}

\section{Cases}

\author{
Amir Saeed (iD) ${ }^{1}$ and Nima Mehdizadegan (iD) ${ }^{2,}{ }^{*}$ \\ ${ }^{1}$ Department of Pediatrics, Division of Pediatric Critical Care, Shiraz University of Medical Sciences, Shiraz, Iran \\ ${ }^{2}$ Cardiovascular Research Center, Shiraz University of Medical Sciences, Shiraz, Iran \\ "Corresponding author: Cardiovascular Research Center, Shiraz University of Medical Sciences, Shiraz, Iran. Email: nmehdizadegan@gmail.com
}

Received 2021 May 21; Revised 2021 August 22; Accepted 2021 August 26.

\begin{abstract}
Introduction: Since the beginning of the coronavirus disease 2019 (COVID-19) outbreak, it was assumed that infection rate in pediatric patients is lower than in adults and that infection is less severe in children than adult patients. Recently, there have been several reports and case series presenting critically-ill children with COVID-19, but still, severe hypotension is rare in pediatric patients with COVID-19.

Case Presentation: We describe three pediatric cases with COVID-19 who presented with multi-system organ failure and severe hypotension treated with the guidance of the parameters of an invasive continuous hemodynamic monitoring device. We also compare their parameters with few articles on pediatric sepsis parameters.

Conclusions: Although we usually start the treatment of hypotensive pediatric patients with hydration and epinephrine as an inotrope, in our cases, we required a different treatment plan according to the hemodynamic monitoring parameters, which indicates the value of the utilization of these devices in pediatric intensive care units
\end{abstract}

Keywords: COVID-19, Multisystem Inflammatory Syndrome in Children (MIS-C), Invasive Hemodynamic Monitoring, Hypotension, Pediatric Intensive Care Unit

\section{Introduction}

In February 2020, the World Health Organization (WHO) declared coronavirus disease 2019 (COVID-19) as a pandemic. This disease was first seen in Wuhan, China, with a group of people who had pneumonia with an unknown cause, and then coronavirus was detected in the respiratory samples of these patients (1) and named the virus severe acute coronavirus 2 (SARS-CoV-2) (2).

The first published data focused on adults as the rate of infection in children was low, and the infected had a milder disease. Although myocardial involvement was reported from the beginning of the pandemic in adult patients, it was believed that pediatric patients are infected with a milder disease. Nonetheless, after a while cases of critically-ill children with COVID-19 who presented with Kawasaki-like symptoms were reported; thus, the WHO set a new definition as "multisystem inflammatory syndrome in children (MIS-C)” (3).
According to the definition, MIS-C is seen in children and adolescents aged up to 19 years, with a history of $>3$ days fever, evidence of COVID-19 (positive RT-PCR or serology) or contact with positive cases of COVID-19, and two of the following diagnostic criteria: mucocutaneous symptoms (e.g., rash or bilateral non-purulent conjunctivitis), cardiovascular disorders (hypotension), myocardial dysfunction (according to ECHO findings or elevated troponin/NT-proBNP), hematology abnormalities (increased PT, PTT, and d-Dimers), acute gastrointestinal symptoms (diarrhea, vomiting, or abdominal pain), and laboratory data indicating inflammation (e.g., increased ESR, C-reactive protein, or procalcitonin), and with the exclusion of bacterial sepsis, toxic shock syndrome, Kawasaki disease, and other diseases (3).

Hypotension is seen in severe cases of MIS-C; myocardial involvement due to direct viral invasion, acidosis, hypoxemia, pulmonary hypertension, cytokine storm, or septic shock resulting in hypotension are some supposed eti- 
ologies for this condition (4).

To approach a pediatric patient with septic shock; generally, clinical assessment according to extremity temperature, capillary refill time, and pulses and pulse pressure has been recommended to differentiate "warm" versus "cold" shock. Warm shock is defined as normal or increased cardiac output (CO) and low systemic vascular resistance (SVR), which is most often seen in adult patients. Cold shock is described with low CO and increased SVR (5), and according to the type of shock, different strategies for resuscitation are used (e.g., vasopressors for warm shock and inotropes for cold shock) (6), but in the latest guideline issued by Surviving Sepsis Campaign recommended using advanced hemodynamic parameters (if available), in addition to bedside clinical variables to guide the resuscitation of children with septic shock (7).

The monitoring of hemodynamic status was performed by an arterial line in the femoral artery, and the catheter of central venous line was inserted into the internal jugular or subclavian vein. Then, they were linked to pulse contour cardiac output (PiCCO), which is an invasive monitoring device. Pulse Contour Cardiac Output uses a combination of two methods for progressive hemodynamic and volumetric monitoring as follows: transpulmonary thermodilution for volumetric quantities of preload and C.O and pulse contour analysis to run constant C.O, cardiac index (CI), SVR, MAP, pulse pressure variation (PPV), stoke volume variation (SVV), etc. (8).

Although it is assumed that children contract a milder disease, still our knowledge about the aspects of this disease, especially the severe forms, is lacking. Herein, we described the cases of three girls with COVID-19 presented with multi-system inflammatory syndrome and severe hypotension, who were treated successfully with the guidance of parameters derived from a continuous invasive hemodynamic monitoring device.

\section{Case Presentation}

\subsection{Case 1}

A10-year-old previously healthy girl was referred to the emergency room (ER) of her local town's hospital due to prolonged fever, diarrhea, and lethargy. A week before hospital admission, she had fever and abdominal pain and received some medications, but her condition did not improve, and three days before admission, she developed diarrhea and her condition aggravated. Thus, she was taken to the hospital. In the ER, her vital signs were as follows: blood pressure (BP): 90/40, heart rate(HR):135, and respiratory rate (RR): 32 . On physical exam, she had bilateral non purulent conjunctivitis, cervical lymphadenopathy, strawberry tongue, and erythema of the hands. Thus, with the diagnosis of atypical Kawasaki disease, she was moved to our pediatric intensive care unit at Nemazee Hospital, in Shiraz (Nemazee Hospital is a tertiary hospital with 750 beds that is located in south of Iran and has an 18-bed medical PICU and a 9-bed surgery PICU). On arrival, her vital signs were: BP:55/30, HR:158, RR:35, and Glasgow coma scale (GCS):7/15. She was intubated and inotrope was started, and RT-PCR for COVID-19 with nasopharyngeal swab was sent for her, the result of which was positive. Laboratory data is listed in Table 1. Her clinical criteria fulfilled the criteria of MIS-C (Figure 1).

Echocardiography findings indicated borderline left ventricle function and dilated inferior vena cava (IVC) (it was performed while high-dose inotrope was infusing).

Arterial and central venous lines were inserted and connected to the PiCCO. According to the hemodynamic data (Table 2), inotropes (dopamine and epinephrine) were increased, and vasoconstrictor was started; in addition to parenteral hydrocortisone, ascorbic acid, and thiamine were administered (HAT protocol). Fortunately, the patient responded well, the inotrope was tapered and discontinued, and she was discharged in an acceptable state.

\subsection{Case 2}

A13-year-old girl was taken to the ER with the complaint of diarrhea, abdominal pain, and fever; three days before admission she presented with fever and abdominal pain, and on the day of admission, she presented with watery diarrhea. Her parents gave history that she had had up to 10 times watery diarrhea in the last hour, and she was taken to the hospital due to fecal incontinence. On arrival at the ER, vital signs were: BP: 110/65, HR: 152, RR: 22, and temperature: $40^{\circ} \mathrm{C}$. She had GCS:13/15 and weak central pulses, so the infusion of dopamine was started, but due to the worsening of her condition (GCS dropped to 11 and BP to 90), she was intubated and transferred to the PICU (in ER, RT-PCR for COVID-19 with nasopharyngeal swab was taken, which showed positive results, and chest CT scan was done that was in favor of COVID-19).

Laboratory data (Table 1) and clinical signs and symptoms were in favor of MIS-C. In the PICU, C.V catheter was inserted, and epinephrine infusion was added. Echocardiogram revealsed poor left ventricle systolic function and borderline right ventricle systolic function with dilated IVC.

For constant hemodynamic observation, arterial line was placed into the femoral artery and connected to the PiCCO. According to low SVR, norepinephrine was started (HAT also was started) and titrated to acceptable MAP, and 


\begin{tabular}{|c|c|c|c|}
\hline & Patient\# 1 & Patient\# 2 & Patient\#3 \\
\hline Ferritin (ng/mL), M: 22.81 - 275, F: 4.63 - 204 & $>2000$ & 539 & \\
\hline COVID realtime PCR & Positive & Positive & Positive \\
\hline Typical chest CT finding & Patchy infiltration & Bilateral patchy ground glass & Diffuse and bilateral opacities \\
\hline White blood cells (count/mL) & 25900 & 3300 & 22000 \\
\hline Lymphocyte count & 950 & 260 & 1100 \\
\hline Procalcitonin, $\leq 0.3$ & 14.2 & 89.9 & 1.8 \\
\hline C-reactive protein, $<6(\mathrm{mg} / \mathrm{L})$ & 150 & 105 & 30 \\
\hline Creatine phosphokinase $(\mathrm{U} / \mathrm{L}), \mathrm{M}:<171, \mathrm{~F}:<145$ & 322 & 2470 & 264 \\
\hline Lactate dehydrogenase $(\mathrm{U} / \mathrm{L}),<480$ & 664 & 1240 & 1350 \\
\hline $\operatorname{Troponin}(\mathrm{ng} / \mathrm{mL}),<19$ & 62.5 & 1163 & 450 \\
\hline D-Dimer $(\mathbf{n g} / \mathbf{m L}),<500$ & 5193 & 2754 & 638 \\
\hline Total bilirubin, $0.1-1.2$ & 0.9 & 0.6 & 0.2 \\
\hline Direct bilirubin, $<0.3$ & 0.5 & 0.2 & 0.1 \\
\hline Aspartate transaminase $(\mathrm{U} / \mathrm{L}), \mathrm{M}:<37, \mathrm{~F}:<31$ & 55 & 114 & 97 \\
\hline Alanine aminotransferase $(\mathrm{U} / \mathrm{L}), \mathrm{M}:<41, \mathrm{~F}:<31$ & 37 & 42 & 13 \\
\hline Albumin & 2.8 & 2.9 & 2.5 \\
\hline Blood urea nitrogen $(\mathrm{mg} / \mathrm{dL}), 8$ - 20 & 24 & 30 & 53 \\
\hline Creatinine, M: 0.8 - 1.3, F: 0.6 - 1.2 & 0.8 & 1 & 4.8 \\
\hline Pt/INR & $17.1 / 1.27$ & 24.1/1.79 & $17.9 / 1.33$ \\
\hline Ptt & 32 & 31.9 & 35 \\
\hline Blood culture & Negative & Negative & Negative \\
\hline ESR & 78 & 54 & 48 \\
\hline Fibrinogen $(200$ - 400) & 426 & 411 & 580 \\
\hline Stool culture/urine culture & Negative & Negative & Negative \\
\hline $\mathrm{SCVO}_{2}$ & 78 & 91 & 68 \\
\hline
\end{tabular}

\begin{tabular}{lccc}
\hline Table 2. The Initial PiCCO Parameters & & & \\
\hline Normal Range & Patient\#1 & Patient\#2 & Patient\#3 \\
\hline Sys BP $(\mathbf{m m H g})$ & 72 & 77 & 72 \\
\hline Dia BP $(\mathbf{m m H g})$ & 43 & 42 & 42 \\
\hline MAP $(\mathbf{m m H g})$ & 53 & 55 & 53 \\
CI $\left(\mathbf{L} / \mathbf{m i n} / \mathbf{m}_{2}\right)$ & 4.39 & 4.34 & 2.6 \\
\hline ITBI & 979 & 680 & 500 \\
ELWI $(\mathbf{c c} / \mathbf{k g})(\mathbf{3}-\mathbf{7})$ & 14 & 6 & 11 \\
\hline GEDI $\left(\mathbf{c c} / \mathbf{m}^{2}\right)(\mathbf{6 8 0}-\mathbf{8 0 0})$ & 420 & 340 & 332 \\
SVRI $(\mathbf{1 7 0 0}-\mathbf{2 4 0 0})$ & 690 & 530 & 2240 \\
\hline PPV $(\%)(\mathbf{0}-\mathbf{1 0})$ & 9 & 12 & 11 \\
SSV $(\%)(\mathbf{0}-\mathbf{1 0})$ & 11 & 17 & 22 \\
PVPI $(\mathbf{1}-\mathbf{3})$ & 3.9 & 2.2 & 1.9 \\
\hline
\end{tabular}

Abbreviations: CI, cardiac index; CE, cerebral edema; EVLWI, extravascular lung water index; MAP, mean arterial pressure; SVRI, systemic vascular resistance index; SVV, stroke volume variation. 


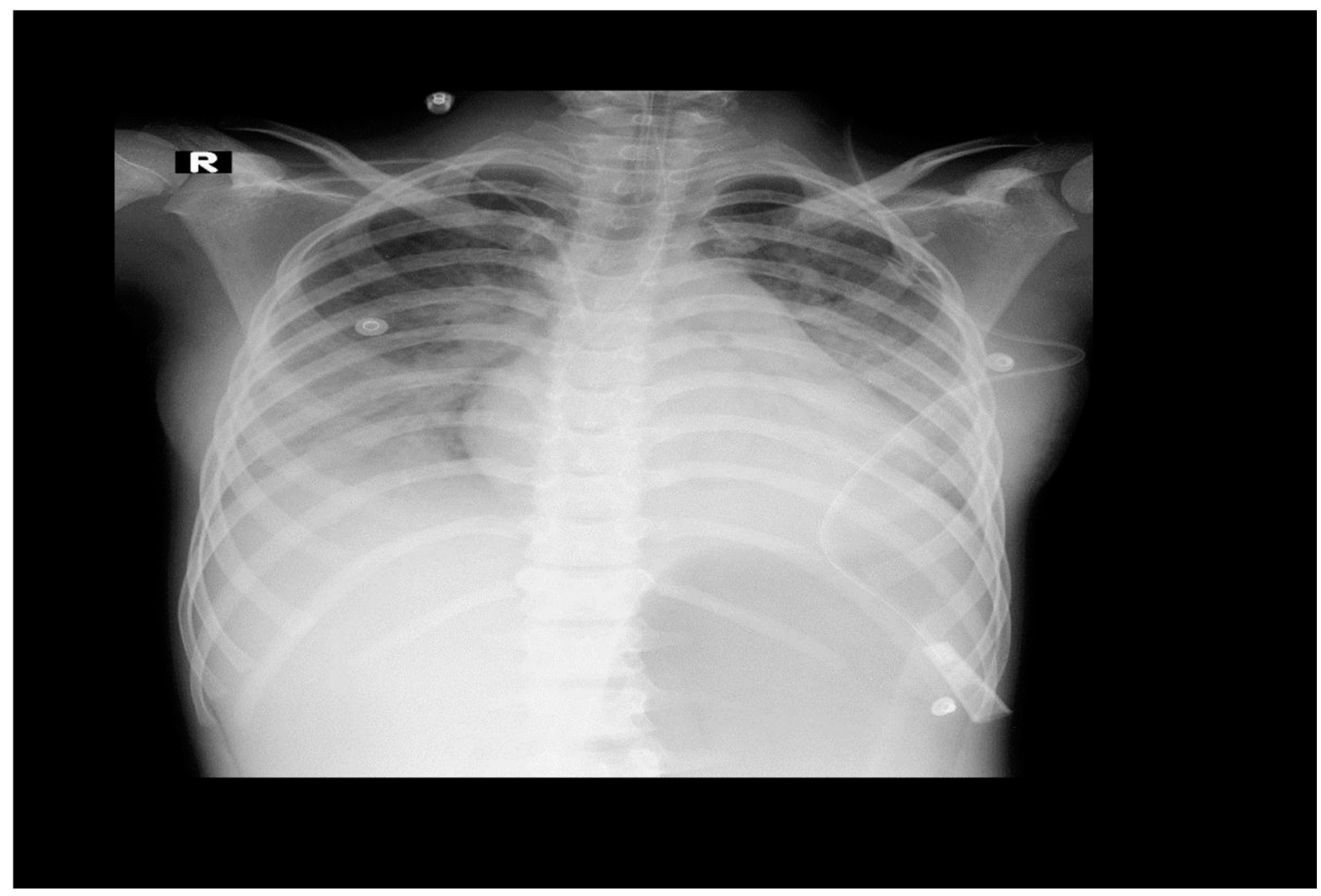

Figure 1. Chest X-ray of patient \#1

then vasopressin was added (the parameters are listed in Table 2).

Gradually, dopamine and epinephrine were tapered to discontinuation on day two, followed by norepinephrine and vasopressin. On the fifth day, she only received 0.2 $\mu \mathrm{g} / \mathrm{kg} / \mathrm{min}$ norepinephrine infusion, but due to severe ARDS, still, she needed mechanical ventilation till the 12th day that she was extubated and tolerated oxygen via nonrebreathing mask, and three days later she was transferred to the ward with a stable and acceptable condition.

\subsection{Case 3}

An 8-year-old girl was taken to the ER due to prolonged fever (four days), convulsion, and decreased level of consciousness (LOC). She also had a previous history of asthma.

On arrival to the ER: GCS:9/15, BP:85/40, HR:138, and RR:42 (laboratory data are listed in Table 1). The echocardiogram revealed fair left ventricle systolic function and moderate right pleural effusion. In the ER, her BP decreased to 55/20; thus, inotrope (dopamine) was started, and the pa- tient was intubated and transferred to the PICU (RT-PCR for COVID-19 was positive).

In the PICU, she was connected to PiCCO with both arterial and C.V catheters; according to its data (Table 2), hydration and inotrope(epinephrine) were started. The inotrope was titrated, and the patient was extubated after four days. On the fifth day, inotrope was discontinued and the patient was discharged on the seventh day.

\section{Discussion}

In this report, we described successful treatment of three children with MIS-C and severe shock with the guidance of PICCO monitoring. This device defines our options for choosing inotropes or hydration for successful treatment.

Patients admitted to ICUs are at risk or have single or multiple organ failure. In such cases, we can use the traditional method: "HR, BP, central venous $\mathrm{O} 2$ saturation, central venous pressure (CVP), and urine output" or use the non-invasive or less invasive methods to direct our treatment (8). 
Pulse contour cardiac output is a less invasive method with significant value when its variables are added to clinical variable in the right way and at the right time, and the patient has regular sinus rhythm and is fully sedated under controlled mechanical ventilation $(6,8,9)$. In pediatric patients, intensivists typically treat septic shock with aggressive fluid administration and achieving the therapeutic goal of CI: $3.3-6.0 \mathrm{~L} / \mathrm{min} / \mathrm{m}^{2}$ (CI is generally low in pediatric septic shock with high SVR); in contrast to adult patients that have low SVR but CO is usually maintained or increased (6-10). As mentioned, we had a different plan for inotrope and hydration for each of our cases.

\section{Acknowledgments}

In our cases, we used the data derived from PiCCO in addition to frequent bedside echocardiography to decide about hydration and inotrope (Figure 1). The parameters of our patients were unique because there are no reported data of hemodynamic parameters in pediatric patients with MIS-C. Therefore, we compared our data with pediatric patients with septic shock. In a study by Deep et al., non-invasive ultrasound cardiac output monitor device (USCOM) was used in 36 pediatric patients with either community-acquired or hospital-acquired sepsis; all of the hospital-acquired septic patients and three of communityacquired ones had low SVR, and the remaining had low cardiac output shock $(\mathrm{CI}<3.3)(12)$. Lee et al. conducted a study with PiCCO in 37 septic patients, the mean CI was $3.75 \pm 1.08$, the mean SVRI was $1327.34 \pm 705.48$, and SVV was $15 \pm 6.5$ (13). Ranjit et al. evaluated fluid refractory pediatric septic shock patients with clinical assessment, bedside echocardiography, and invasive BP monitoring. They found that $56.3 \%$ of the patients had warm shock, $85.5 \%$ had vasodilatory shock on invasive BP, and $29 \%$ initially presented with cold shock (14). Ceneviva et al. reported 50 children with fluid-refractory $(\geq 60 \mathrm{~mL} / \mathrm{kg}$ in the first hour), dopamine-resistant shock. More than half of the patients (58\%) showed a low CO/high SVR state, and $22 \%$ had low CO and low vascular resistance (15). Abdalaziz et al. studied 45 patients with community-acquired septic shock by bedside echocardiography and reported that $82 \%$ of their cases had the echocardiographic criteria of cold septic shock with low normal or low CI $(\leq 3.3$ $\left.\mathrm{L} / \mathrm{m} / \mathrm{m}^{2}\right)$ and normal or high SVRI $\left(\geq 1,600\right.$ dyn-sec $/ \mathrm{cm}^{5}$ $\left./ \mathrm{m}^{2}\right)$, whereas the remaining had the echocardiographic criteria of warm septic shock (high CI $>6 \mathrm{~L} / \mathrm{m} / \mathrm{m}^{2}$ ) with low normal or low SVRI $\left(\leq 800\right.$ dyn-sec $\left./ \mathrm{cm}^{5} / \mathrm{m}^{2}\right)(16)$. Hydration in the management of septic shock is a doubleedged sword; it can correct relative hypovolemia, which is due to capillary leakage, not taking enough fluids, and fluid losses and maintain organ flow (9); on the other hand, overhydration may cause pulmonary edema or increase mortality (17). The patients No. 1 and 2 had dilated IVC and low SVV and PPV; thus, there was no need for hydration; the initial CI was 4.39 in case 1 and case 2's was 4.34, and SVRI was low in both patients (case 1: 690 dynes/seconds $/ \mathrm{cm}^{5}$ vs 530 dynes/seconds $/ \mathrm{cm}^{5}$ in case 2 ). Accordingly, norepinephrine was our first choice and was started and titrated up to $1.6 \mu \mathrm{g} / \mathrm{kg} / \mathrm{min}$ in addition to vasopressin that was titrated to a high dose to taper norepinephrine. Although we used high doses of these vasoconstrictors, neither liver nor renal function impairment was seen in our patients, but the patient No. 3 was volume responsive (increased SVV,PPV) with low CI. Thus, the patient was hydrated, and epinephrine was started as an inotrope. A vasodilatory shock associated with COVID-19 is seen in adult patients, which may be due to the dysfunction of angiotensin-converting enzyme (ACE) resulted from severe pulmonary endothelial damage in COVID-19 patients with acute respiratory distress syndrome (ARDS). Thus, some centers use angiotensin II infusion in vasodilatory shock in COVID-19 patients $(18,19)$, which can be one of the mechanisms used for hypotension in pediatric patients. In the reported articles, PICU intubation rate in pediatric COVID-19 patients was 15 - 47\% (20), while all of our patients were intubated in spite of not complaining of respiratory problem or respiratory symptoms in the first two patients. Only patient number 3 has refractory hypoxemia. $\mathrm{PaO}_{2} / \mathrm{FiO}_{2}$ ratio before intubation in case 1 was 273, and in case 2, it was 220 (but in case 2, 24 hours later it dropped to 112 and 62 on the third day), but patient No. 3 had a low $\mathrm{PaO}_{2} / \mathrm{FiO}_{2}$ ratio (150). On day three, patients No. 1 and 2 needed increased ventilator support, and the condition of case 2 worsened $\left(\mathrm{PaO}_{2} / \mathrm{FiO}_{2}\right.$ ratio: 56$)$. Patient No. 2 needed to increase peak end expiratory pressure up to 15 centimeters of water, and the median peak pressure reached $38 \mathrm{~cm}$ of water. The main problem in all of our patients was refractory hypoxemia. In sum, we cannot solely rely on our traditional shock management that starts with dopamine or epinephrine, instead we should use methods to direct us in choosing the best inotrope or time for hydration. By increasing number of COVID-19 pediatric patients, PiCCO could be used successfully in critically-ill cases complicated with MIS-C for the appropriate management of shock and severe dehydration and judicious inotrope use. Additional studies are required on larger groups and with more accurate devices to provide further parameters to help clinicians in better hemodynamic management of this disease. 


\section{Footnotes}

Authors' Contribution: A.S. planned the study and wrote the manuscript, and submitted the manuscript, and N.M. gathered patients' data and edited the manuscript. All the authors discussed the results and contributed to the final manuscript.

\section{Conflict of Interests: None.}

Ethical Approval: This study was approved by the Ethics Committee of Shiraz University of Medical Sciences with the approval ID: IR.sums.med.rec.1398.336.

Funding/Support: The authors did not receive any specific grant for this research from any funding agency in the public, commercial or not-for-profit sectors.

Informed Consent: Written informed consent was obtained from the parents and sent to the ethics committee.

\section{References}

1. Zhu N, Zhang D, Wang W, Li X, Yang B, Song J, et al. A novel coronavirus from patients with pneumonia in China, 2019. $N$ Engl J Med. 2020;382(8):727-33. doi: 10.1056/NEJMoa2001017. [PubMed: 31978945]. [PubMed Central: PMC7092803].

2. World Health Organization. Novel coronavirus (2019-nCoV): Situation report, 22. Geneva: World Health Organization; 2020.

3. World Health Organization. Multisystem inflammatory syndrome in children and adolescents with COVID-19: scientific brief. World Health Organization; 2020.

4. Chen Q, Xu L, Zhu W, Ge J. Cardiovascular manifestations in severe and critical patients with COVID-19. Clin Cardiol. 2020;43(10):1054. doi: 10.1002/clc.23422. [PubMed: 32667051]. [PubMed Central: PMC7405227].

5. Martin K, Weiss SL. Initial resuscitation and management of pediatric septic shock. Minerva Pediatr. 2015;67(2):141-58. [PubMed: 25604591]. [PubMed Central: PMC4395852].

6. Monnet X, Vaquer S, Anguel N, Jozwiak M, Cipriani F, Richard C, et al. Comparison of pulse contour analysis by Pulsioflex and Vigileo to measure and track changes of cardiac output in critically ill patients. Br J Anaesth. 2015;114(2):235-43. doi: 10.1093/bja/aeu375. [PubMed: 25516277].

7. Weiss SL, Peters MJ, Alhazzani W, Agus MSD, Flori HR, Inwald DP, et al. Surviving sepsis campaign international guidelines for the management of septic shock and sepsis-associated organ dysfunction in children. Pediatr Crit Care Med. 2020;21(2):e52-e106. doi: 10.1097/PCC.0000000000002198. [PubMed:32032273].

8. Huygh J, Peeters Y, Bernards J, Malbrain ML. Hemodynamic monitoring in the critically ill: an overview of current cardiac output monitoring methods. F1000Res. 2016;5. doi: 10.12688/f1000research.8991.1. [PubMed: 28003877]. [PubMed Central: PMC5166586].
9. Smetkin AA, Hussain A, Kuzkov VV, Bjertnaes LJ, Kirov MY. Validation of cardiac output monitoring based on uncalibrated pulse contour analysis vs transpulmonary thermodilution during off-pump coronary artery bypass grafting. Br J Anaesth. 2014;112(6):1024-31. doi: 10.1093/bja/aet489. [PubMed: 24531685].

10. Davis AL, Carcillo JA, Aneja RK, Deymann AJ, Lin JC, Nguyen TC et al. American college of critical care medicine clinical practice parameters for hemodynamic support of pediatric and neonatal septic shock. Crit Care Med. 2017;45(6):1061-93. doi: 10.1097/ccm.0000000000002425. [PubMed: 28509730].

11. Alhazzani W, Moller MH, Arabi YM, Loeb M, Gong MN, Fan E, et al. Surviving sepsis campaign: Guidelines on the management of critically ill adults with coronavirus disease 2019 (covid-19). Intensive Care Med. 2020;46(5):854-87. doi: 10.1007/s00134-020-06022-5. [PubMed: 32222812]. [PubMed Central: PMC7101866].

12. Deep A, Goonasekera CD, Wang Y, Brierley J. Evolution of haemodynamics and outcome of fluid-refractory septic shock in children. Intensive Care Med. 2013;39(9):1602-9. doi: 10.1007/s00134-013-3003-z. [PubMed: 23812341].

13. Lee EP, Hsia SH, Lin JJ, Chan OW, Lee J, Lin CY, et al. Hemodynamic analysis of pediatric septic shock and cardiogenic shock using transpulmonary thermodilution. Biomed Res Int. 2017;2017:3613475. doi: 10.1155/2017/3613475. [PubMed: 28401152]. [PubMed Central: PMC5376469].

14. Ranjit S, Aram G, Kissoon N, Ali MK, Natraj R, Shresti S, et al. Multimodal monitoring for hemodynamic categorization and management of pediatric septic shock: a pilot observational study*. Pediatr Crit Care Med.2014;15(1):e17-26. doi:10.1097/PCC.0b013e3182a5589c. [PubMed: 24196006].

15. Ceneviva G, Paschall JA, Maffei F, Carcillo JA. Hemodynamic support in fluid-refractory pediatric septic shock. Pediatrics. 1998;102(2). e19. doi: 10.1542/peds.102.2.e19. [PubMed: 9685464].

16. Abdalaziz FA, Algebaly HAF, Ismail RI, El-Sherbini SA, Behairy A. The use of bedside echocardiography for measuring cardiac index and systemic vascular resistance in pediatric patients with septic shock. Rev Bras Ter Intensiva. 2018;30(4):460-70. doi: 10.5935/0103-507X.20180067. [PubMed: 30672970]. [PubMed Central: PMC6334480].

17. Maitland K, Kiguli S, Opoka RO, Engoru C, Olupot-Olupot P, Akech SO, et al. Mortality after fluid bolus in African children with severe infection. N Engl J Med. 2011;364(26):2483-95. doi: 10.1056/NEJMoa1101549. [PubMed: 21615299].

18. Chow JH, Mazzeffi MA, McCurdy MT. Angiotensin II for the treatment of COVID-19-related vasodilatory shock. Anesth Analg. 2020;131(1):1025. doi: 10.1213/ANE.0000000000004825. [PubMed: 32209811]. [PubMed Central: PMC7172573].

19. Zangrillo A, Landoni G, Beretta L, Morselli F, Serpa Neto A, Bellomo $\mathrm{R}$, et al. Angiotensin II infusion in COVID-19-associated vasodilatory shock: a case series. Crit Care. 2020;24(1):227. doi: 10.1186/s13054-02002928-0. [PubMed: 32414393]. [PubMed Central: PMC7228670].

20. Chao JY, Derespina KR, Herold BC, Goldman DL, Aldrich M, Weingarten J, et al. Clinical characteristics and outcomes of hospitalized and critically ill children and adolescents with coronavirus disease 2019 at a tertiary care medical center in New York City. J Pediatr. 2020;223:14-19 e2. doi: 10.1016/j.jpeds.2020.05.006. [PubMed: 32407719]. [PubMed Central: PMC7212947]. 